Pecvnia, Monográfico (2008), pp. 205-223

\title{
Fuentes externas de conocimiento y su efecto sobre el esfuerzo innovador en los sectores industriales y de servicios en España
}

Hoy en día, no son los fabricantes los únicos encargados del desarrollo de nuevos productos, procesos o servicios, sino que se hace necesario reconocer la importancia que tienen otros agentes externos a la empresa como fuente de actividades innovadoras. La complejidad y dinamismo de los entornos actuales obliga a las empresas a complementar su base interna de conocimientos con otros procedentes del exterior. Estas circunstancias han llevado a distinguir entre fuentes internas y externas de innovación. Puesto que las primeras han sido suficientemente estudiadas y analizadas en la literatura, el presente trabajo pretende avanzar sobre el

\author{
Gloria Sánchez-González \\ gloria.sanchez@unileon.es \\ Liliana Herrera \\ liliana.herrera@unileon.es \\ Universidad de León \\ Organización de Empresas \\ Campus de Vegazana, s/n \\ 24071 León (España)
}

Fac. de Ciencias Económicas y Empresariales

\begin{abstract}
Manufacturers are currently not the only ones in charge of developing new products, processes or services. Instead, nowadays it is necessary to recognize the importance of other external agents to the organization as sources of innovation activities. The present complex and dynamic environments force organizations to complement their internal knowledge with other sorts of external information. These circumstances have created a distinction between internal and external innovation sources. Given that the first have been studied and analysed thoroughly in the literature, the present study tries to advance in the understanding of the second and its
\end{abstract}


conocimiento de las segundas y sus implicaciones en la actividad innovadora de las empresas. Para ello se ha analizado la influencia de nueve de esas fuentes externas sobre la intensidad total de la actividad innovadora y de las actividades de $1+D$ intramuros y extramuros del conjunto del sector productivo español durante el periodo 2001-2003, llegando a la conclusión de que los clientes son quienes mayor impacto ejercen en los tres casos.

Palabras clave: fuentes externas de innovación, cooperación, intensidad de la actividad innovadora (total, intramuros y extramuros).

\begin{abstract}
implications with regard to the organizations innovation activities. In order to achieve this objective, the present article analyzes the effect of nine external sources on the total innovation activity intensity and also on the internal and external R\&D activities of the whole Spanish productive sector during the period spanning from 2001 to 2003. This study concludes that cooperation with customers had the most significant effect in all of the tree cases.
\end{abstract}

Key words: external innovation sources, cooperation, innovation intensity (total, intramural and extramural).

\section{INTRODUCCIÓN}

El entorno en que se desarrollan las actividades económicas en la mayoría de los países se caracteriza por un gran dinamismo y complejidad. Los cambios bruscos en la tecnología, en los clientes y en los competidores, obligan a las empresas a una continua renovación para sobrevivir y en muchos casos, esos cambios pasan por buscar nuevas formas de hacer las actividades, entre las que se encuentran las actividades innovadoras.

Tradicionalmente, los economistas al estudiar los procesos de innovación partían de la premisa de que los fabricantes de productos eran los desencadenantes de dichos procesos. Frente a esto, los investigadores sobre el cambio tecnológico y organizativo han demostrado que asumir el hecho de que el fabricante es la única fuente de innovaciones limita mucho la visión del proceso innovador (von Hippel 1988). Partiendo de esta idea, se reconoce la importancia que tiene para la actividad innovadora contar con fuentes de información y conocimiento localizadas fuera de la organización. En este contexto, a lo largo de la literatura se ha reconocido que los rendimientos de la empresa dependerán en gran medida de su habilidad para encontrar, absorber y manejar de forma productiva esas fuentes (Cohen y Levinthal 1990).

Aunque recientemente estas ideas son ampliamente aceptadas, la conveniencia de complementar la base de conocimiento interno de la empresa con fuentes externas fue reconocida inicialmente 
por Alfred Marshall en 1925, al resaltar en su trabajo la importancia que tiene para en el progreso económico que las empresas desarrollen externalidades positivas a través de una organización basada en el mercado (Marshall 1925: 335). Esas fuentes externas pueden tener orígenes muy diversos, desde usuarios hasta suministradores de componentes y materiales u otros agentes implicados en el proceso de innovación (von Hippel 1988).

Figura 1: Fuentes internas y externas origen de actividades innovadoras

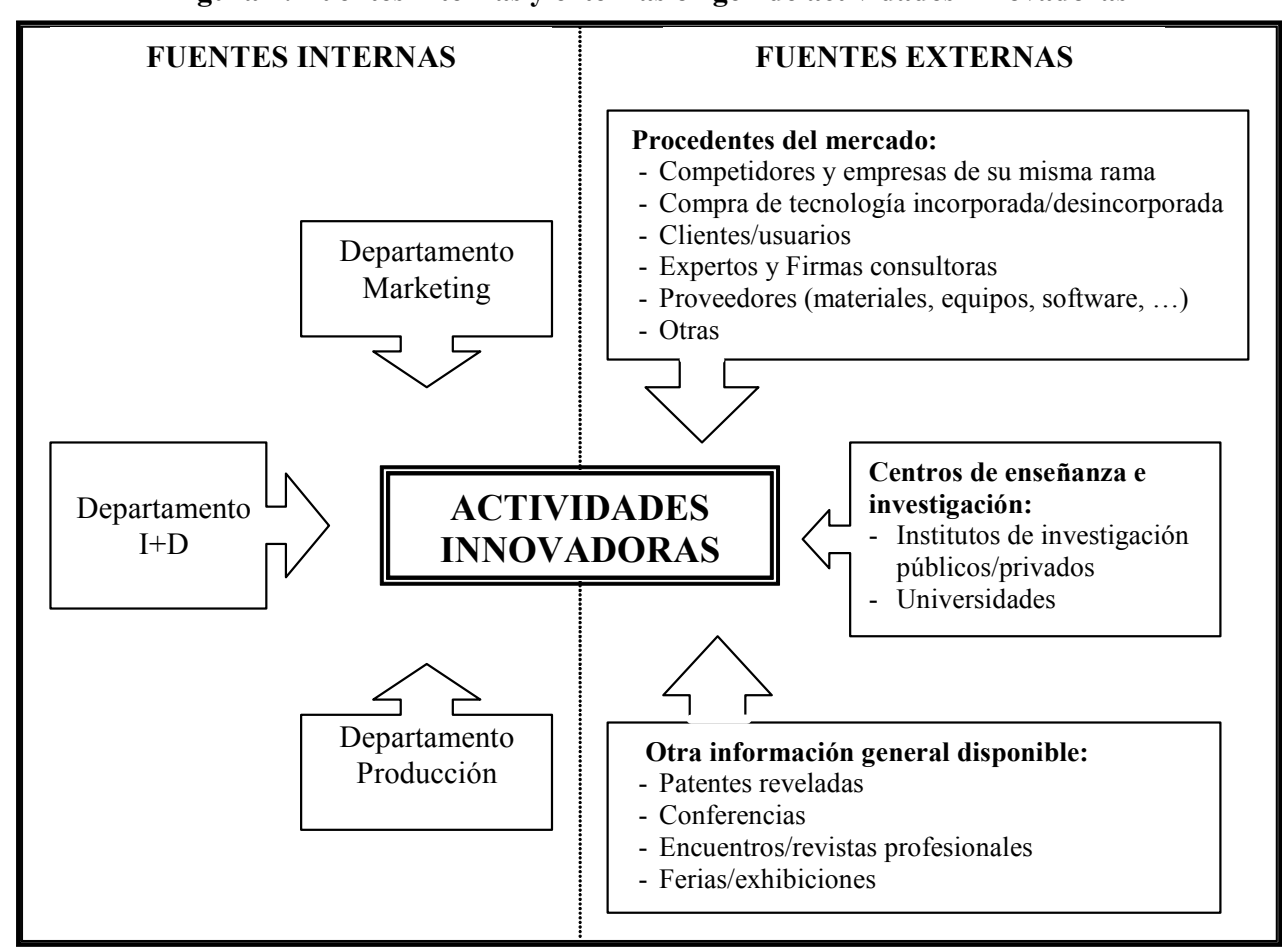

Fuente: Elaboración propia.

En este sentido el departamento de I+D de cualquier empresa no constituye la única fuente de actividades innovadoras. Con carácter general las fuentes de innovación se dividen en dos tipos: internas versus externas, algunas de las cuales se detallan en la Figura 1. Las primeras hacen referencia al conjunto de actividades innovadoras que se llevan a cabo dentro de la organización, especialmente en los departamentos de I+D, marketing y producción. Las segundas abarcan: a) fuentes procedentes del mercado, como competidores o empresas de su misma rama, compra de tecnología incorporada o desincorporada, clientes o consumidores, expertos y firmas consultoras, proveedores de equipos, materiales, componentes y 
software, centros tecnológicos, laboratorios comerciales o empresas de I+D, etc.), b) centros de enseñanza o de investigación entre los que se encuentran los institutos públicos y privados de investigación y las universidades y c) información general disponible relativa a patentes reveladas, conferencias, encuentros y revistas profesionales así como ferias y exhibiciones (von Hippel 1988; OCDE 1997).

A diferencia de los enfoques sobre el cambio tecnológico y el crecimiento económico, en la literatura sobre dirección estratégica, concretamente en el Enfoque basado en los Recursos (EBR) (Penrose 1959; Rumelt 1984; Teece 1984; Wernerfelt 1984; Barney 1991; Peteraf 1993), se ha demostrado que las relaciones con agentes externos a la empresa constituyen un importante recurso en el actual marco competitivo, especialmente en lo que se refiere al desarrollo de nuevos productos y procesos. De acuerdo con esta perspectiva, las variaciones en los rendimientos de las empresas que compiten en una misma industria podrían explicarse a partir de las diferencias en su dotación y utilización de recursos (Barney 1986a, 1986b, 1991, 2001; Peteraf 1993; Wernerfelt 1984), recursos entre los que se encuentran las fuentes de innovación a las que recurre la empresa.

Dentro de este marco, se considera que las empresas que cuentan con recursos valiosos, escasos, insustituibles e inimitables podrán alcanzar y mantener en el tiempo una posición ventajosa respecto a sus competidores (Barney 1995: 56). Por tanto, no resulta difícil entender que entre la gran diversidad de recursos que posee una empresa, tangibles e intangibles, sean éstos últimos los que ofrecen mayores posibilidades de convertirse en fuente de ventaja competitiva (Ray, Barney y Muhanna 2004). Estos intangibles pueden acumularse en la empresa y su origen podría encontrarse en las relaciones que ésta mantiene con agentes externos en materia de innovación. Su elevado componente tácito y su complejidad social (Dierickx y Cool 1989; Peteraf 1993), hacen precisamente que estos recursos sean difícilmente identificables y/o reproducibles por cualquier competidor.

Este hecho resulta evidente en el caso concreto de los clientes como fuente de innovación. Así, por ejemplo, es relativamente fácil obtener del mercado información acerca de las necesidades explícitas que tienen los usuarios respecto a los productos ofrecidos por la empresa, mediante encuestas u otras técnicas tradicionales de investigación de mercados que pueden ser utilizadas igualmente por los rivales. Esta información no constituirá por tanto una fuente de ventaja competitiva. 
Sin embargo, si la organización permite a sus usuarios colaborar en los procesos de innovación y desarrollo de nuevos productos, será capaz de descubrir necesidades de las que, en muchos casos, ni los propios usuarios son conscientes. Esas relaciones difícilmente serán imitadas por competidores, con lo que la empresa disfrutará de datos muy valiosos sobre los que diseñar una estrategia competitiva exitosa.

Estos planteamientos, en los que se reconoce el papel que juegan los usuarios en el desarrollo de actividades innovadoras, han dado pie al surgimiento de una literatura centrada en las innovaciones promovidas por la demanda (entre otros estudios: Rosenberg 1976; von Hippel 1976, 1977a, 1986; Lundvall 1988; Herstatt y von Hippel 1992; Shah 2000; Franke y Shah 2003; Lüthje 2004; Lüthje et al. 2005). Concretamente, se hace necesario reconocer el papel destacado que juegan los usuarios en la actividad innovadora, al menos en ciertas industrias.

Lo anterior deja claro que las empresas difieren en el tipo de fuente de innovación que emplean y en consecuencia, en su esfuerzo innovador. Por tanto, conocer la fuente de la actividad innovadora resultará determinante para mejorar su ventaja competitiva.

De igual modo, se ha contrastado en la literatura que los sectores también se diferencian en sus fuentes de innovación y estas desigualdades podrían atribuir a la intensidad en la cooperación con distintos agentes externos. La cooperación en materia de innovación viene justificada por múltiples motivos, como por ejemplo la constante reducción de los ciclos de vida de los productos que obliga a tener que aumentar paulatinamente los costes para mantenerse a la cabeza de la frontera tecnológica o la creciente complejidad e interdisciplinariedad de la innovación muy vinculado con cuestiones de aprendizaje o incluso por cuestiones estratégicas, como se vio anteriormente.

Por todo ello, el objetivo del presente trabajo es medir el efecto de algunas fuentes externas de innovación sobre la actividad innovadora del conjunto de sectores productivos españoles. Dado que la actividad innovadora difiere ampliamente entre sectores, se pretende determinar si estas diferencias pueden ser explicadas a partir del grado de cooperación de las empresas con distintos agentes externos.

De entre la variedad de fuentes externas de innovación en este estudio se han considerado nueve, que son: 1) empresas del mismo grupo, 2) clientes, 3) proveedores, 4) competidores y empresas de la misma rama, 5) expertos y firmas consultoras, 6) laboratorios comerciales 
o empresas de I+D, 7) universidades, 8) organismos públicos de I+D y 9) centros tecnológicos. De igual manera el efecto de estas fuentes se ha estimado empleando distintos indicadores de la actividad innovadora y concretamente tres: la intensidad total en actividades innovadoras, la intensidad en I+D intramuros y la intensidad en I+D extramuros. Esta última distinción se realiza con el fin de obtener conclusiones respecto al efecto de las distintas fuentes externas de innovación sobre el nivel de desarrollo interno o externo de tecnología.

Para cumplir con estos propósitos, en el segundo apartado se comenta la situación de la actividad innovadora en relación a veinte sectores productivos de la economía española durante el período 20012003, junto con un análisis descriptivo de los niveles de cooperación en relación a las mencionadas nueve fuentes. En el tercer apartado, mediante tres modelos de regresión múltiple, se identifican las fuentes de innovación que más inciden sobre la intensidad total en actividades innovadoras, así como en I+D intramuros y extramuros. Por último, en el apartado cuarto, se recogen los resultados y discusión del estudio empírico y finalmente, en el apartado quinto se presentan las conclusiones.

\section{LA ACTIVIDAD INNOVADORA Y LAS FUENTES EXTERNAS DE INNOVACIÓN EN EL SECTOR PRODUCTIVO ESPAÑOL}

Ampliamente se sabe que la innovación difiere entre sectores en términos de las características, fuentes, actores involucrados, niveles de apropiación, bases de conocimiento, intensidad y organización de la actividad innovadora. A esta conclusión se ha llegado gracias a un importante cuerpo de evidencia empírica que ha encontrado diferencias sectoriales en estos aspectos [ver Malerba (2005) para una discusión más amplia].

En el caso de España y para el periodo 2001-2003 (ver Tabla 1 ), puede apreciarse que los sectores manufactureros que registraron mayores niveles de intensidad en actividades innovadoras fueron el sector de Maquinaria y Material de Transporte, el sector del Textil, Confección, Cuero y Calzado y el de Productos Químicos. Por el lado de los servicios destacaron las Inmobiliarias y Servicios a Empresas junto con el sector de las Comunicaciones. En cuanto a los sectores que menos esfuerzo realizaron en innovación en relación con su cifra de negocio se encontraron el de Construcción y el de Energía y Agua por el lado de las industrias, y los de 
Intermediación Financiera y Comercio y Hostelería por el lado de los servicios.

Atendiendo a los esfuerzos en I+D intramuros destacaron nuevamente, en cuanto a manufacturas, los sectores de Productos Químicos y de Maquinaria y Material de Transporte cuyos esfuerzos en actividades de $1+D$ representaron un $1,52 \%$ y un $0,92 \%$ sobre su cifra de negocio respectivamente $\mathrm{y}$, en cuanto a los servicios, los de Inmobiliarias y Servicios a Empresas, con un 1,26\% sobre su cifra de negocio. Los sectores que ejercieron una menor intensidad en actividades de $I+D$ internas durante dicho periodo fueron de nuevo la industria de la Construcción, con un $0,05 \%$, y los servicios de Comercio y Hostelería y el de Transportes y Almacenamiento, con unos valores del $0,03 \%$ y $0,08 \%$, respectivamente sobre su cifra de negocios.

Por último, destacar que comparativamente con la intensidad en actividades de I+D intramuros, la intensidad en actividades de I+D extramuros fue considerablemente menor, con carácter general, en todos los sectores, exceptuando los casos de la Construcción (un 0,06\% en I+D externa frente a un $0,05 \%$ en I+D interna) y el de los servicios de Comercio y Hostelería (un $0,2 \%$ en I+D externa frente a un 0,03\% en I+D interna). En este apartado, los sectores con mayores intensidades resultaron ser exactamente los mismos que en el caso anterior para las empresas manufactureras, Maquinaria y Material de Transporte y Productos Químicos, mientras que en los sectores de servicios destacaron de nuevo las Inmobiliarias y Servicios a Empresa y las Comunicaciones. Los sectores que menos esfuerzos dedicaron a la I+D extramuros fueron el de Alimentación, Bebidas y Tabaco dentro del primer grupo, y los de Transportes y Almacenamiento junto con el de Servicios Públicos, Sociales y Colectivos en el segundo grupo.

Por otro lado, numerosos estudios indican que los niveles de cooperación son muy distintos en función del tipo de sector (Hladik 1985; Link y Bauer 1989; Hagedoorn 1993; Wang 1994). En este contexto, no cabe duda que la consideración de estas diferencias sectoriales se convierte en un aspecto importante a la hora de evaluar los efectos de la cooperación con distintas fuentes de innovación externas a la empresa sobre la actividad innovadora del sector productivo. 


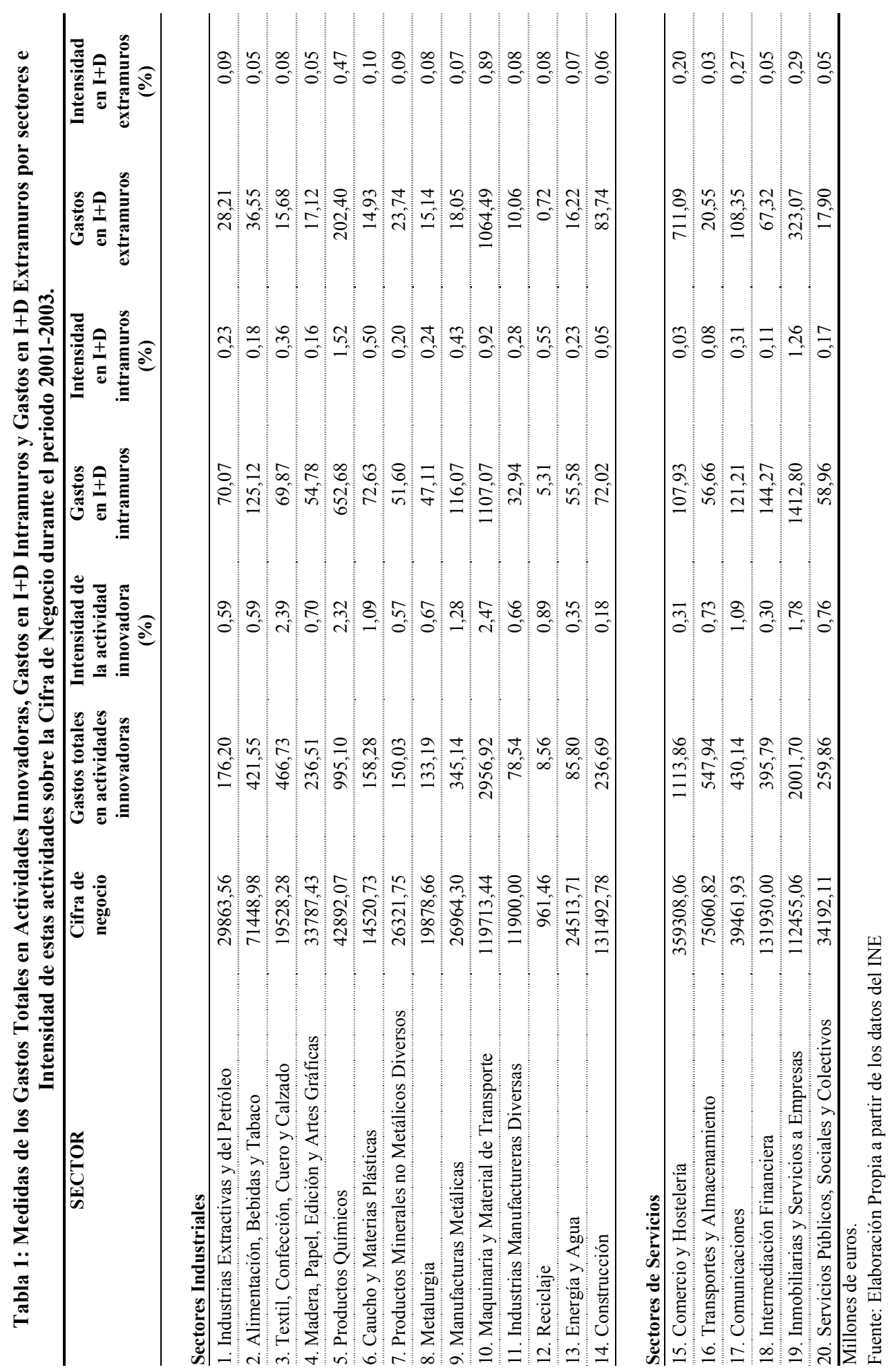


Así, para la realización de este estudio se han considerado nueve fuentes externas a la empresa con las que es posible cooperar en materia de innovación (ver Tabla 2). Concretamente en España y considerando únicamente empresas innovadoras o con innovaciones en curso o no exitosas (EIN), cabe destacar que un $40 \%$ de dichas empresas cooperó con proveedores, un $26,9 \%$ lo hizo con universidades y un $19,2 \%$ cooperó con expertos y firmas consultoras. Esas 5.710 empresas que cooperaron en innovación en el periodo 2001-2003 representaron el 15\% del total del sector productivo español.

Tabla 2: Cooperación en innovación en el periodo 2001-2003 según tipo de interlocutor

\begin{tabular}{lrr}
\hline Empresas EIN que han cooperado en innovación en 2001-2003 con: & Total & $\mathbf{\%}$ \\
\cline { 2 - 4 } & $\mathbf{5 . 7 1 0}$ & $\mathbf{1 0 0}$ \\
\hline - Otras empresas de su mismo grupo & 835 & 14,6 \\
\hline - Clientes & 733 & 12,8 \\
\hline - Proveedores & 2.283 & 40,0 \\
\hline - Competidores y otras empresas de su misma rama & 668 & 11,7 \\
\hline - Expertos y firmas consultoras & 1.095 & 19,2 \\
\hline - Laboratorios comerciales o empresas de I+D & 454 & 8,0 \\
\hline - Universidades & 1.534 & 26,9 \\
\hline - Organismos públicos de I+D & 673 & 11,8 \\
\hline - Centros tecnológicos & 900 & 15,8 \\
\hline
\end{tabular}

* Una empresa puede cooperar con más de una unidad.

Fuente: INE (2003)

En cuanto a la modalidad de cooperación por ramas de actividad y considerando el total de empresas de cada sector (ver Tabla 3) se desprende que, con carácter general, el sector que claramente sobresalió fue el de Productos Químicos, en el que un $18,56 \%$ de sus empresas realizaron algún tipo de cooperación en esta materia. Con menores niveles de cooperación, entre el $10 \%$ y el $12 \%$, se encontraron las industrias Metalúrgicas, de Reciclaje y de Maquinaria y Material de Transporte. En cuanto a los servicios, es la Intermediación Financiera el que presentó el porcentaje más alto de empresas que cooperaron en innovación $(10,6 \%)$.

Si se tiene en cuenta el tipo de agente con el que se realizó la cooperación destacaron las universidades, especialmente en el caso de Productos Químicos (8,38\%) y en el de Energía y Agua (6,71\%). Así mismo, también fue importante la cooperación con expertos y firmas consultoras en el sector del Reciclaje $(6,66 \%)$ y la cooperación con centros tecnológicos en la industria Metalúrgica (5,79\%). 


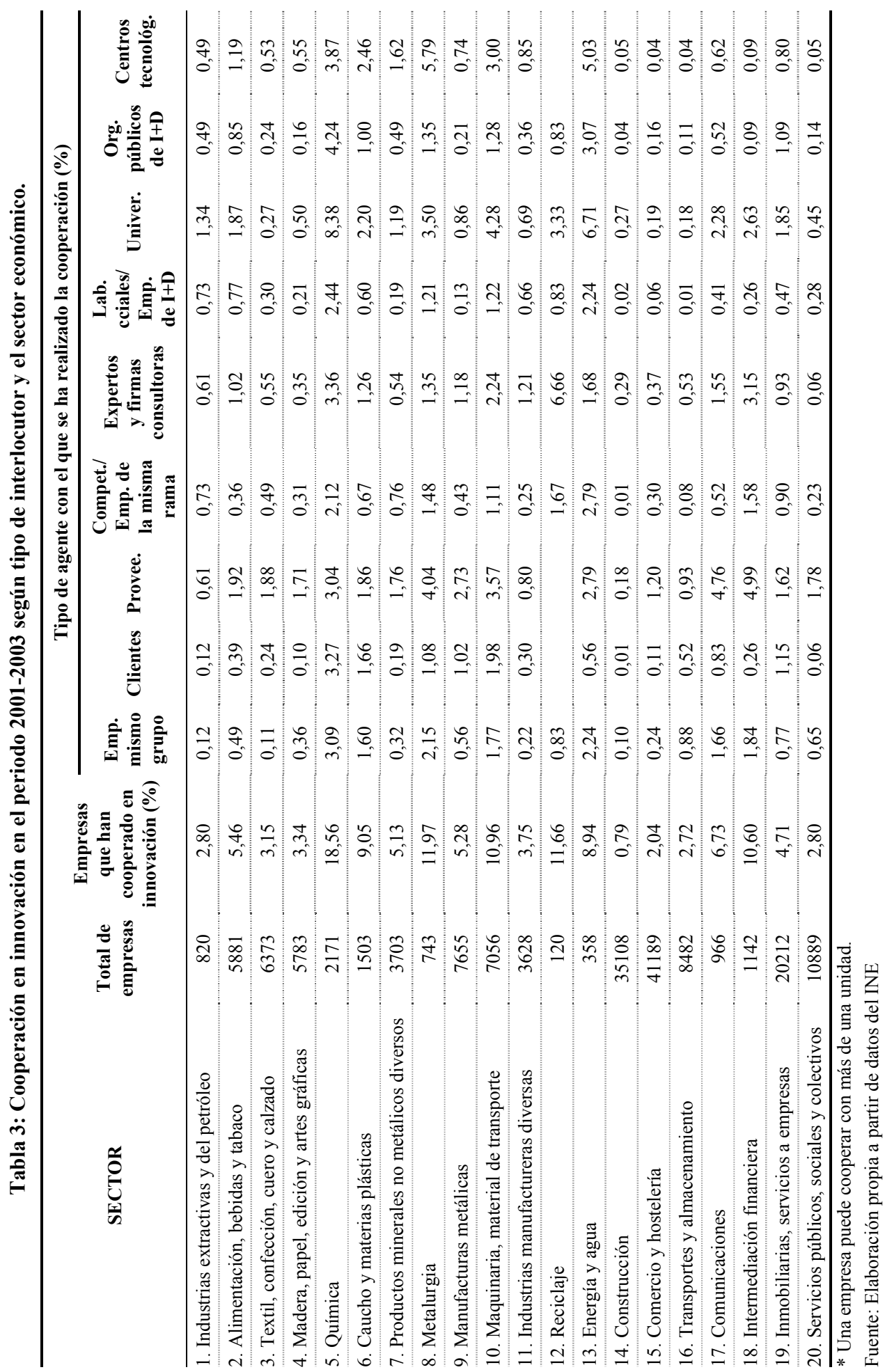




\section{LA METODOLOGÍA}

\subsection{Datos}

Este estudio se ha realizado a partir de una muestra de veinte sectores (ver Tablas 2 y 3 ) que integran el conjunto de empresas, manufactureras y de servicios, de la economía española. Los datos se han recopilado de la encuesta sobre Innovación Tecnológica de las Empresas (INE 2003) y corresponden al periodo 2001-2003. Conviene apuntar que esta encuesta ha sido diseñada para facilitar información sobre la estructura del proceso de innovación (I+D y otras actividades innovadoras) y permite mostrar las relaciones entre dicho proceso y la estrategia tecnológica de las empresas, los factores que favorecen (o dificultan) su capacidad para innovar y el rendimiento económico de las empresas.

\subsection{El modelo}

En este trabajo se pretende analizar cuantitativamente el efecto que ejercen nueve tipos de cooperación en innovación sobre la intensidad de la actividad innovadora del sector productivo español. El objetivo es identificar, de entre las nueve formas de cooperación en innovación aquí consideradas, cual/es tienen un impacto significativo sobre la intensidad de la actividad innovadora y si dicha cooperación aumenta o disminuye esa intensidad.

Para cumplir con ese objetivo se formula la siguiente ecuación de regresión para el encontraste de tres modelos cuyas variables independientes recogen los nueve tipos de cooperación anteriormente mencionados y cuyas variables dependientes son: la intensidad total en actividades innovadoras ${ }^{1}$ (modelo 1 ), la intensidad en $I+D$ intramuros (modelo 2 ) y la intensidad en I+D extramuros (modelo 3 ):

$$
\mathrm{Y}_{\mathrm{i}}=\alpha+\beta_{1} \mathrm{X}_{1 \mathrm{i}}+\beta_{2} \mathrm{X}_{2 \mathrm{i}}+\beta_{3} \mathrm{X}_{3 \mathrm{i}}+\beta_{4} \mathrm{X}_{4 \mathrm{i}}+\beta_{5} \mathrm{X}_{5 \mathrm{i}}+\beta_{6} \mathrm{X}_{6 \mathrm{i}}+\beta_{7} \mathrm{X}_{7 \mathrm{i}}+\beta_{8} \mathrm{X}_{8 \mathrm{i}}+\beta_{9} \mathrm{X}_{9 \mathrm{i}}+\varepsilon_{\mathrm{i}}
$$

\footnotetext{
Se habla de intensidad total en actividades innovadoras porque en ella no sólo se tienen en consideración la I+D interna y externa, sino también el esfuerzo realizado en otras actividades como la formación, la adquisición de otros conocimientos externos, la introducción de la innovación en el mercado, el diseño y otros preparativos, la adquisición de maquinaria y equipos, etc. (INE 2003). Sin embargo, al ser la I+D interna y externa las actividades que representan la mayor parte del gasto en innovación (el 65\% del total de gasto en actividades para la innovación), son las más interesantes para realizar un análisis individualizado.
} 
Donde:

$\mathrm{Y}_{1 \ldots 3}=$ intensidad de la actividad innovadora ${ }^{2}$

$\alpha=$ constante de la regresión

$\beta_{\mathrm{i}}=$ pendiente correspondientes a las diferentes variables independientes

$\mathrm{X}_{1}=$ porcentaje de empresas que han cooperado en innovación con otras empresas del mismo grupo $\left(\mathrm{COP}_{1}\right)$

$\mathrm{X}_{2}=$ porcentaje de empresas que han cooperado en innovación con clientes $\left(\mathrm{COP}_{2}\right)$

$\mathrm{X}_{3}=$ porcentaje de empresas que han cooperado en innovación con proveedores $\left(\mathrm{COP}_{3}\right)$

$\mathrm{X}_{4}=$ porcentaje de empresas que han cooperado en innovación con competidores y empresas de la misma rama $\left(\mathrm{COP}_{4}\right)$

$\mathrm{X}_{5}=$ porcentaje de empresas que han cooperado en innovación con expertos y firmas consultoras $\left(\mathrm{COP}_{5}\right)$

$\mathrm{X}_{6}=$ porcentaje de empresas que han cooperado en innovación con laboratorios comerciales/empresas de I+D $\left(\mathrm{COP}_{6}\right)$

$\mathrm{X}_{7}=$ porcentaje de empresas que han cooperado en innovación con universidades $\left(\mathrm{COP}_{7}\right)$

$\mathrm{X}_{8}=$ porcentaje de empresas que han cooperado en innovación con organismos públicos de I+D $\left(\mathrm{COP}_{8}\right)$

$\mathrm{X}_{9}=$ porcentaje de empresas que han cooperado en innovación con centros tecnológicos $\left(\mathrm{COP}_{9}\right)$

$\varepsilon_{\mathrm{i}}=$ término de error de la regresión múltiple

El signo resultante para los diferentes coeficientes $\beta_{\mathrm{i}}$ que acompañan a las variables explicativas relativas al tipo de cooperación indica que:

2 En el modelo 1 la variable dependiente, $Y_{1}$, se refiere a la intensidad de la actividad innovadora (INTEN), medida como: (Gastos en Actividades Innovadoras / Cifra de negocio) $x$ 100. En el modelo 2 la variable dependiente, $Y_{2}$, se refiere a la intensidad de la actividad innovadora realizada a través de I+D intramruos (INTEN ID INT), medida como: (Gastos en I+D interna / Cifra de negocio) $\times 100$. $Y$ en el modelo 3 la variable dependiente, $Y_{3}$, se refiere a la intensidad de la actividad innovadora realizada a través de I+D extramuros (INTEN_ID_EX), medida como: (Gastos en I+D externa / Cifra de negocio) x 100 . 
Si $\beta_{1 \ldots 9}>0$ : el tipo de cooperación aumenta la intensidad de la actividad innovadora

Si $\beta_{1 \ldots 9}<0$ : el tipo de cooperación reduce la intensidad de la actividad innovadora

\section{RESULTADOS DEL ANÁLISIS ESTADÍSTICO Y DISCUSIÓN}

En vista de los resultados recogidos en la Tabla 4 la validez de los modelos es considerablemente alta en los tres casos. En el modelo 1 , el coeficiente de determinación $\left(\mathrm{R}^{2}\right)$ está muy próximo al 0,8 , lo que indica que cerca del $80 \%$ de la variación en la intensidad total de la actividad innovadora del sector productivo se explica por la cooperación tecnológica. De igual modo, el conjunto de variables que reflejan las diferentes formas de cooperación explican el $89,5 \%$ de la variación experimentada en la intensidad de la actividad innovadora realizada a través de I+D intramuros (modelo 2 ) y el $77,3 \%$ de la variación experimentada en la intensidad de la actividad innovadora realizada a través de I+D extramuros (modelo 3 ). Lo anterior demuestra que los modelos propuestos son adecuados para explicar el efecto que el tipo de cooperación en innovación tiene sobre la intensidad de la actividad innovadora en el conjunto de la economía española.

Tabla 4: Coeficientes estandarizados de las variables independientes y validez de los modelos de Regresión Múltiple

\begin{tabular}{|c|c|c|c|}
\hline Tipo de cooperación & $\begin{array}{c}\text { MODELO } 1 \\
\text { Intensidad } \\
\text { actividad } \\
\text { innovadora }\end{array}$ & $\begin{array}{c}\text { MODELO } 2 \\
\text { Intensidad } \\
\text { en I+D } \\
\text { intramuros }\end{array}$ & $\begin{array}{c}\text { MODELO } 3 \\
\text { Intensidad } \\
\text { en I+D } \\
\text { extramuros }\end{array}$ \\
\hline - (Constantes) & 0,144 & 0,023 & 0,091 \\
\hline - Cooperación con empresas del mismo grupo & $-0,731$ & $-0,303$ & $-0,212$ \\
\hline - Cooperación con clientes & $1,587 * * *$ & $0,704 * * *$ & $0,343 * *$ \\
\hline - Cooperación con proveedores & $0,401 * *$ & 0,074 & 0,010 \\
\hline - Cooperación con competidores y empresas de la misma rama & 0,890 & $0,439 *$ & $-0,005$ \\
\hline - Cooperación con expertos y firmas consultoras & $-0,488$ & $-0,129$ & $-0,191$ \\
\hline - Cooperación con laboratorios comerciales/empresas de I+D & 1,420 & 0,185 & 0,131 \\
\hline - Cooperación con universidades & $-0,426$ & $-0,100$ & $0,328 * *$ \\
\hline - Cooperación con organismos públicos de $\mathrm{I}+\mathrm{D}$ & $-0,234$ & 0,077 & $-0,550 * *$ \\
\hline - Cooperación con centros tecnológicos & $-0,293$ & $-0,126^{*}$ & $-0,047$ \\
\hline \multicolumn{4}{|l|}{ Estadístico $\mathrm{R}^{2}$ de los Modelos } \\
\hline $\mathrm{R}^{2}$ & 0,796 & 0,895 & 0,773 \\
\hline
\end{tabular}


En cuanto al efecto de los distintos tipos de cooperación se encontró que las relaciones con clientes resultaron tener una influencia significativa y positiva en los tres modelos. El hecho de que los coeficientes estandarizados sean en los tres casos positivos, implica que su efecto se materializa en un aumento de la intensidad innovadora cuanto mayor sea el porcentaje de empresas que cooperan con sus clientes sobre el total de empresas.

Otro resultado interesante es que además en el primer y segundo modelo, esa cooperación con clientes es la variable independiente que presenta los coeficientes más altos y por tanto, esa forma de cooperación es la principal determinante del aumento de la intensidad de la actividad innovadora y de la intensidad de la actividad innovadora realizada a través de I+D intramuros.

En principio estos resultados son contradictorios con las previsiones iniciales, puesto que la literatura sobre este tema generalmente demuestra que la utilización de clientes durante el proceso innovador reduce los gastos de innovación (Thomke y Nimgade 1998; Herstatt y von Hippel 1992; Jeppesen 2002, 2005; Chan y Lee 2004; Henkel y von Hippel 2004; von Hippel 2005). Sin embargo, este no es el único estudio que obtiene este tipo de resultados (Lillien et al. 2002). Ahora bien, la explicación en este caso concreto puede deberse a que los datos han sido tratados agregadamente por sectores productivos mientras que en el resto de estudios el análisis se ha realizado sobre casos de empresas puntuales. Habría que ver por tanto, si este hecho tiene alguna trascendencia sobre el signo resultante.

También se debe considerar que los clientes no tienen obligación de conocer las características ni el funcionamiento de los procesos productivos de la empresa, de modo que las mejoras que proponen no tendrían por que considerar estos aspectos, pudiendo implicar importantes modificaciones para adaptar el proceso a los nuevos diseños. En esta misma línea, hay que considerar que para que el cliente pueda aportar ideas valiosas es necesario darle una cierta formación y herramientas que le ayuden en el proceso de generación y diseño de nuevos productos, aspectos que también implican costes.

Pero en definitiva, lo que este resultado deja patente es que el hecho de cooperar con clientes demuestra un mayor interés por desarrollar actividades innovadoras y por tanto, por dedicar mayores esfuerzos a estas tareas. 
En cuanto a la cooperación con proveedores, ésta incide sobre la intensidad de la actividad innovadora en general y, al igual que con los clientes, lo hace positivamente, pudiendo explicarse este hecho con los mismos argumentos que en el caso anterior.

Por su parte la cooperación con competidores y empresas de la misma rama, incide significativamente sobre la intensidad de la I+D interna y el signo de esa relación también se presenta positivo. El razonamiento que cabría realizar en este caso es que teniendo en cuenta que de este modo la empresa comparte ciertos conocimientos con sus competidores, simultáneamente tendrá que buscar la forma de preservar una posición ventajosa respecto a ellos y una vía será incrementando sus esfuerzos en actividades de I+D interna, por ejemplo para desarrollando nuevos procesos productivos más eficientes.

En lo referente a la cooperación con entidades públicas, éstas no presentan una influencia significativa en el caso de la intensidad total en la actividad innovadora. No obstante, los resultados cambian cuando se consideran medidas más restrictivas del esfuerzo innovador como la intensidad en I+D intramuros y extramuros.

En el primer caso, la cooperación con centros tecnológicos reduce significativamente el esfuerzo innovador interno de los sectores productivos, lo que presumiblemente puede ser interpretado como un traslado de las actividades de investigación y desarrollo a estos centros.

En el caso de la intensidad en I+D extramuros, la cooperación con universidades tiene una influencia positiva y significativa, de manera que estimula el crecimiento de las inversiones en tecnología externa. Este resultado difiere del propósito de muchos programas públicos de apoyo a la innovación cuyo objetivo es estimular el desarrollo interno, a diferencia del externo, de innovaciones a través de la cooperación universidad-empresa.

Finalmente, la cooperación con organismos públicos de I+D tiene una influencia negativa y significativa sobre la intensidad en investigación y desarrollo extramuros. Un resultado que se ajusta a la finalidad de la cooperación con estos organismos la cual, en principio, al ser subvencionada por el gobierno, intenta reducir el gasto externo en I+D.

Frente a todo esto, la cooperación con expertos y firmas consultoras, así como con laboratorios comerciales o empresas de I+D ha resultado no tener una influencia significativa en ninguno de los modelos 
y por lo tanto, la cooperación con estos agentes no afecta a la actividad innovadora del sector.

\section{CONCLUSIONES}

El objetivo fundamental de este trabajo ha sido determinar la influencia que puede ejercer la cooperación con distintos agentes externos a la empresa sobre la intensidad total de la actividad innovadora, la intensidad de en I+D intramuros y la intensidad en I+D extramuros en el sector productivo español. Para ello se han considerado un total de veinte sectores y la cooperación con nueve tipos distintos de fuentes de innovación: 1) empresas del mismo grupo, 2) clientes, 3) proveedores, 4) competidores y empresas de la misma rama, 5) expertos y firmas consultoras, 6) laboratorios comerciales o empresas de I+D, 7) universidades, 8) organismos públicos de I+D y 9) centros tecnológicos.

De este modo ha quedado patente que la cooperación en materia de innovación no es un fenómeno puntual, tal y como pone de manifiesto el hecho de que un $15 \%$ del total de empresas españolas hayan cooperado de una forma u otra. Sin embargo, al mismo tiempo también se deduce que, pese a que la literatura demuestra que la cooperación con clientes en actividades innovadoras reduce los costes de desarrollo del proceso innovador, además de otras muchas ventajas, en el caso español estas cuestiones no están siendo tenidas en cuenta, dado el reducido porcentaje que representa este tipo de cooperación $(12,8 \%)$ en relación a otros como proveedores $(40 \%)$ o universidades $(26,9 \%)$.

Es más, del análisis estadístico se desprende que es precisamente la cooperación con clientes la que determina de forma más significativa tanto la intensidad de las actividades innovadoras en general, como de las de I+D intramuros y extramuros, en particular. Además, puesto que el sentido de esa relación es positivo en los tres casos, significa que aquellos sectores que más cooperan con clientes son al mismo tiempo los que más esfuerzos realizan en actividades innovadoras. En cierta forma, podría decirse, que la cooperación con clientes demuestra ser un indicador de la importancia que la empresa otorga a la innovación, puesto que cuanto mayor sea esta cooperación, mayores recursos se destinan a las actividades innovadoras. 
De acuerdo con estos resultados las empresas españolas deberían fomentar e implantar políticas estratégicas que potencien la cooperación con agentes externos a la organización en actividades de innovación y especialmente, en relación a sus clientes.

En último lugar cabe señalar que este trabajo podría completarse mediante un análisis longitudinal más amplio con el objetivo de conocer mejor la evolución de estas diferentes formas de cooperación y su efecto en la transformación de los sectores productivos españoles.

\section{BIBLIOGRAFÍA}

BARNEY, J.B. (1986a) "Strategic Factor Markets: Expectations, Luck, and Business Strategy", Management Science, Vol. 32, 10, pp. 1231-1241.

- (1986b) "Organizational Culture: Can It Be a Source of Sustained Competitive Advantage?", Academy of Management Review, Vol. 11, 3, pp. 656-665.

- (1991) "Firm Resources and Sustained Competitive Advantage", Journal of Management, Vol. 17, 1, pp. 99-120.

- (1995) "Looking Inside for Competitive Advantage", Academy of Management Executive, Vol. 9, pp. 49-61.

- (2001) "Is the Resource-Based 'View' a Useful Perspective for Strategic Management Research? Yes", Academy of Management Review, Vol. 26, 1, pp. 41-56.

ChAN, T.Y. \& J.F. LeE (2004) "A Comparative Study of Online User Communities Involvement In Product Innovation and Development", $13^{\text {th }}$ International Conference on Management of Technology, IAMOT.

COHEN, W.M. \& D.A. LeVINTHAL (1989) "Innovation and Learning: The Two Faces of R\&D", The Economic Journal, Vol. 99, 397, pp. 569-596.

DIERICKX, I. \& K. COOL (1989) "Asset Stock Accumulation and Sustainability of Competitive Advantage", Management Science, Vol. 35, 12, pp. 1504-1511.

FRANKE, N. \& S. SHAH (2003) "How Communities Support Innovative Activities: An Exploration of Assistance and Sharing Among End-Users", Research Policy, Vol. 32, 1, pp. 157-178. 
HAGEDOORN, J. (1993) "Understanding the Rationale of Strategic Technology Partnering: Interorganizational Models of Cooperation and Sectorial Differences", Strategic Management Journal, Vol. 14, 5, pp. 371-385.

HENKEL, J. \& E. VON HIPPEL (2004) "Welfare Implications of User Innovation", The Journal of Technology Transfer, Vol. 30, 1-2, pp. 73-87.

HeRSTATt, C. \& E. von HIPPEL (1992) "FROM EXPERIENCE: Developing New Product Concepts Via the Lead User Method: A Case Study in a 'LowTech' Field", Journal of Product Innovation Management, Vol. 9, 3, pp. 213-221.

HLADIK, K. (1985) International Joint Ventures. Lexington, Massachusetts: Lexington Books.

INSTITUTO NACIONAL DE ESTADÍSTICA (2003) Encuesta sobre Innovación Tecnológica de las Empresas 2003. Madrid: INE.

JePPESEN, L.B. (2002) "Making Consumer Knowledge Available and Useful. The Case of the Computer Games", DRUID Working Paper $n^{\circ} 01-10,2^{\mathrm{a}}$ version.

- (2005) "User Toolkits for Innovation: Consumers Support Each Other", Journal of Product Innovation Management, Vol. 22, 4, pp. 347-362.

LILIEN, G.L. et al. (2002) "Performance Assessment of the Lead User IdeaGeneration Process for New Product Development", Management Science, Vol. 48, 8, pp. 1042-1059.

LINK, A. \& L. BAUER (1989) Cooperative Research in U.S. Manufacturing. Lexintong, Massachussetts: Lexington Books.

LUNDVALL, B.A. (1988) "Innovation as an Interactive Process: from User Producer Interaction to the National System of Innovation". G. Dosı et al. (eds.) Technical Change and Economic Theory. Londres, UK.: Printer Publishers, pp. 349-369.

LÜTHJE, C. (2004) "Characteristics of Innovating Users in a Consumer Goods Field: An Empirical Study of Sport-Related Product Consumers", Technovation, Vol. 24, 9, pp. 683-695.

-, C. HeRstatt \& E. VON HipPel (2005) "User-Innovators and 'Local' Information: The Case of Mountain Biking", Research Policy, Vol. 34, 6, pp. 951-965.

MALERBA, F. (2005) "Sectoral Systems: How and Why Innovation Differs Across Sectors". J. FAgerberg, D. MOWERY \& R. NeLSON (Eds.) The Oxford Handbook of Innovation. New York: Oxford University Press.

MARSHALL, A. (1925) Principles of Economics. Londres, UK.: Macmillan. 
OCDE (1997) OECD Proposed Guidelines for Collecting and Interpreting Technological Innovation Data - Oslo Manual. Paris: OECD Publications Service.

PEnROSE, E.T. (1959) The Theory of the Growth of the Firm. New York: Wiley.

Peteraf, M.A. (1993) "The Cornerstones of Competitive Advantage: A Resource Based-View", Strategic Management Journal, Vol. 14, 3, pp. 179-191.

RAY, G.; J.B. BARNEY \& W.A. MUHANNA (2004) "Capabilities, Business Processes, and Competitive Advantage: Choosing the Dependent Variable in Empirical Tests of the Resource-Based View", Strategic Management Journal, Vol. 25, 1, pp. 23-37.

Rosenberg, N. (1976) Perspectives on Technology. Cambridge: Cambridge University Press.

SHAH, S. (2000) "Sources and Patterns of Innovation in a Consumer Products Field: Innovations in Sporting Equipment", MIT Sloan School of Management, Working Paper $n^{\circ} 4105$.

TEECE, D.J. (1984) "Economic Analysis and Strategic Management", California Management Review, Vol. 26, 3, pp. 87-110.

Thomke, S. \& A. Nimgade (1998) "Note on Lead User Research", Harvard Business School. Note: 9-699-014.

VON HIPPEL, E. (1976) "The Dominant Role of Users in the Scientific Instrument Innovation Process", Research Policy, Vol. 5, 2, pp. 212-239.

- (1977a) "The Dominant Role of the User in Semiconductor and Electronic Subassembly Process Innovation", IEEE Transactions on Engineering Management EM-24, pp. 60-71.

- (1986) "Lead Users: A Source of Novel Product Concepts", Management Science, Vol. 32, 7, pp. 791-805.

- (1998) "Economics of Product Development by Users: The Impact of 'sticky' Local Information", Management Science, Vol. 44, 5, pp. 629644.

- (2005) Democratizing Innovation. Cambridge, MA.: MIT Press.

WANG, J.C. (1994) "Cooperative Research in a Newly Industrialized Country: Taiwan", Research Policy, Vol. 23, pp. 697-711.

WERnERFELT, B. (1984) "A Resource Base View of the Firm", Strategic Management Journal, Vol. 5, pp. 171-180. 